\title{
FACTORES QUE INFLUYERON EN LA DECISIÓN DE CREAR EMPRESAS EN NEIVA: TREINTA AÑOS PENSANDO EN EL FUTURO*
}

\section{FACTORS THAT INFLUENCED THE DECISION TO CREATE COMPANIES IN NEIVA: THIRTY YEARS THINKING ABOUT THE FUTURE}

\author{
Pedro Luis Huergo Tobar**, Jorge Enrique Cuéllar Sánchez ${ }^{\star \star \star}$, Julio Roberto Jaime Salas ${ }^{\star \star \star \star ~}$
}

\begin{abstract}
Resumen
El contexto nacional influye en las empresas desde su misma creación impactando de manera directa en la economía nacional. En esta investigación las empresas fueron estudiadas desde su contexto, tomando el pensamiento individual del empresario; se analizaron los factores más influyentes en la toma de decisión de crear empresas en Neiva por el periodo 1973 a 2003. La investigación buscó sistematizar la información a partir del análisis estadístico y datos cuantitativos utilizando técnicas descriptivas. El universo estuvo conformado por 24.201 firmas, se tuvo en cuenta los factores individual, familiar, social y económico. Se concluye de manera general que los agentes generadores fueron: Ofrecer un mejor futuro a su descendencia, voluntad de prestar un servicio a la comunidad, preparación académica enfocada a cultura emprendedora, asegurar su futuro e indiscutiblemente, identificación de oportunidades de negocio.
\end{abstract}

Palabras clave: Decisión, empleo, emprendimiento, empresa, familia.

\section{Abstract}

The national context of business environment is affected by specific conditions that influence its creation, positively impacting the national economy. In this research the companies were studied from their context, taking into account the individual thought of the entrepreneur; the most influential factors in making decision to create companies in Neiva for the period 1979 to 2003 were analyzed. The research aims to systematize the information, based on statistical analysis and quantitative data, using descriptive techniques. The target population of study is made up of 24.201 enterprises, taking into account the individual, family, social and economic factors. It was conclude, in general, that the most outstanding agents were: the

Artículo recibido: 21/05/2013 Aprobado: 01/08/2013

* Este artículo es la síntesis del proyecto de Investigación Factores que influyeron en la decisión de crear empresas en Neiva en el periodo comprendido entre los años 1973 a 2003, aprobado en Convocatoria CONADI de la Universidad Cooperativa de Colombia, seccional Neiva. Proyecto propuesto inicialmente por GRUPO DE INVESTIGACIÓN START - UP: Investigador principal: Armando de Jesús Herrera, coinvestigadores: Susana Vargas C. y Alexander Pastrana Monje; desarrollado y finalizada por el grupo de investigación VISIONARIOS perteneciente a la facultad de ciencias contables, económicas y administrativas de la sede Neiva.

** Magíster en educación. Especialista en proyectos de inversión. Profesor del programa de Contaduría Pública y Administración de Empresas en la Universidad Cooperativa de Colombia sede Neiva. Director del Grupo de investigación VISIONARIOS perteneciente a la facultad de Ciencias Contables y Administrativas y registrado en COLCIENCIAS desde el año 2009. Email:pedro.huergo@campusucc.edu.co

*** Magíster en Contabilidad y auditoría. Docente Universidad Cooperativa de Colombia Sede Neiva. Email: jorgeenriquecuellar@gmail.com

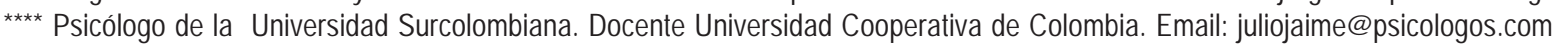


desire to provide a better future for their offspring, the desire to provide a service to the community, academic preparation focused on entrepreneurial culture, the desire to ensure their future, and unquestionably, identifying business opportunities.

Key words: decision, employment, entrepreneurship, business, family.

\section{Introducción}

Todos los factores que rodean al empresario y a su organización tanto internos como externos son importantes para identificar las razones reales que movieron al emprendedor a tomar la decisión de crear empresa. Morales y Roig (2005) proponen que las condiciones específicas que influyen en la creación de empresas se ven afectadas por el contexto general del entorno nacional, es decir, aquellas características de la economía, factores demográficas y culturales de la sociedad en donde se desarrolla la actividad empresarial tales como acceso a financiación, políticas y programas gubernamentales, educación, formación, normas sociales, y las condiciones consideradas individuales y familiares, pueden tener gran impacto sobre este proceso.

Pero según Julien (2008) otros aspectos a tener en cuenta son los factores denominados económicos tales como las oportunidades tomadas aquí o allá y las desarrolladas gradualmente que se presentan al aprovechar las condiciones generales nacionales del entorno y las condiciones específicas que influyen igualmente en la decisión de crear una empresa. Para Toledano y Urbano (2008) una oportunidad de negocio es una idea atractiva duradera y adecuada que en un momento específico que toma la forma de un producto o servicio dirigido a un comprador 0 consumidor.

Lo anterior es lo base para lo que denomina proceso emprendedor se logra con el desarrollo de las habilidades 0 las potencialidades de cada individuo, sus percepciones 0 análisis y aprovechamiento de oportunidades del medio en que se halla inmerso integrando funciones, actividades y acciones asociadas con la explotación de oportunidades; su capacidad e inclinación de aprovecharlas tiene dos aspectos: el primero se encuentra relacionado con el entrenamiento o la habilidad y el segundo con la motivación e interés en el proyecto de empresa, a esto se le ha denominado factores de creación (Toledano \& Urbano, 2008, p. 45 ). El determinar el grado de importancia de estos factores en la mente del empresario en la creación de empresas en la ciudad de Neiva, constituye el objeto de estudio de este artículo.

Para el desarrollo del estudio se miró la parte del pensamiento individual del empresario y se conservó el análisis crítico dado por Gibb (1994) referentes a los componentes del modelo de carrera empresarial, cuyos factores son los individuales, sociales y económicos, buscando abrir una luz al pensamiento examinador frente al tema y de la misma forma dar visos que planteen opciones a los gobernantes, para el desarrollo de políticas, encaminadas a buscar crecimiento de empresas en el ámbito municipal, departamental y nacional.

En la medida que muchos tengan acceso al trabajo, a los ingresos y/o al empleo, las desigualdades serán cada vez menores; de allí que se requiera con urgencia de personas dedicadas a crear fuentes permanentes y rentables de empleo mediante la materialización de sus espacios de trabajo en beneficio de todos (Pallares, Romero, \& Herrera, 2005), de allí la necesidad de que surjan nuevos agentes económicos capaces de crear y distribuir riqueza, generando nuevas rentas y así, mejorar el nivel de vida y bienestar en general (Llopis, F. 2008).

En la historia reciente del sector empresarial colombiano se viene presentando un proceso de deterioro de la actividad económica en la ciudad de Neiva. En el país esto se evidencia en la caída del PIB de 1978 al 2000 $(3,90 \% \text { a } 1,66 \%)^{1}$ y que claramente se ve reflejado en la Gráfica 1; de esta manera se demuestra la relación directa proporcional, entre el Producto Interno Bruto y los Niveles de Pobreza.

1. Subdirector del DNP. Datos suministrados por el Gobierno Nacional de Colombia a través de su Departamento de Planeación Nacional. Documento No. 261 del 9 de julio de 2004. 
En lo que respecta a Neiva, la crisis económica se ve reflejada en la alta proporción de la población pobre que alcanza niveles del $26,20 \%$ según el índice necesidades básicas insatisfechas (NBI), la tasa de desempleo (TD) se encuentra en un $16.2 \%$ y la tasa de subempleo (TS) en un $42.9 \%$. El fundamento más claro se basa en los bajos niveles de la actividad empresarial. Esto se puede advertir del alto coeficiente de natalidad/mortalidad empresarial, que desde la década del setenta ha venido en aumento con un ligero desaceleramiento en los años 2.001 y 2.003 .

El fundamento más claro se basa en los bajos niveles de la actividad empresarial, esto se puede advertir del alto coeficiente de natalidad/mortalidad empresarial, que desde la década del 70 ha venido en aumento, con un ligero desaceleramiento en los años 2.001 y 2.003. Ver fórmula a continuación:

Número de empresas inscritas $\mathrm{CCN}$ año $\mathrm{A}$

Coeficiente mortalidad $=$

Número de empresas no activas año subsiguiente $B$

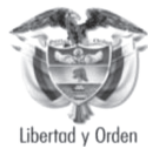

Departamento Nacional de Planeación República de Colombia

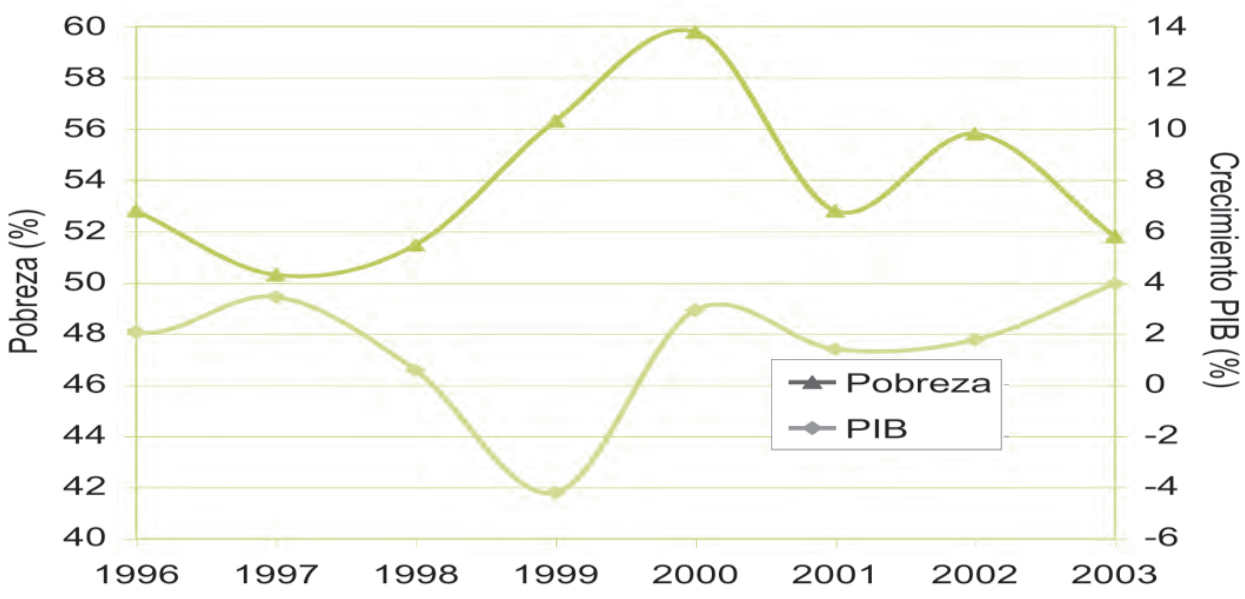

Fuente: (Leivobich, 2005)²

Gráfica 1. Relación entre pobreza y el crecimiento del PIB.

Si se analiza el coeficiente que relaciona la natalidad con mortalidad de empresas en Neiva se aprecia, en el Cuadro 1 , que este coeficiente ${ }^{3}$ para la década del setenta, era de 7,2; para los ochenta, 8,1; para la década del noventa, 7,7 y para el año 2000 era de 6,7, pero en parti- cular el año 2003, se situó en 9,4. De esta gráfica se desprende que mientras diez empresas ingresan al mercado, siete salen en el mismo periodo de la década del setenta. Este mismo coeficiente se aumenta a 8,1 en la década de los ochenta, presentando una leve disminución en la

2. Los desafíos institucionales para reducir la pobreza en Colombia, Seminario medición de la pobreza y estrategias para reducirla, (p. 6).

3. El coeficiente es un indicador construido para grupo de investigación de emprendimiento de la UCC sede Neiva. Este describe la relación del número de empresas que mueren por diez que nacen en el mismo periodo. 
década del noventa al situarse en 7,7 . Finalmente se sitúa en 6,5 en el año 2000, con un preocupante 9,4 en el año 2003. Ver Gráfica 2, y Cuadro 1 a continuación.

Este comportamiento explica en gran parte la situación de pobreza y desempleo que vive hoy la ciudad de Neiva, si tenemos en cuenta que existe una alta correlación entre actividad empresarial y crecimiento económico tal como lo señala Varela (2001), quien afirma que hay una tendencia que indica, que los países con mayor nivel de actividad empresarial presentan los mayores niveles de crecimiento en su producto nacional bruto y en su nivel de empleo.

El anterior postulado se ve reflejado en el bajo crecimiento económico que vivió la economía colombiana desde la segunda mitad de la década de los noventa, cuando se presentó una tendencia descendente desde 1.995 al 2.003 en su PIB ${ }^{4}$ y sus consecuencias especialmente manifestadas en el desempleo empresarial y en la generación de emple $0^{5}$. Algunas explicaciones sobre el lento y diferenciado crecimiento del PIB y del apreciable aumento de las importaciones entre 1992 y 1997 las da Colmenares (2003) quien afirma que no es tanto resultado de los cambios en las regulaciones sobre comercio
Cuadro 1. Coeficiente de Natalidad/Mortalidad en empresas de Neiva.

\begin{tabular}{|lc|}
\hline Décadas & Coeficiente \\
\hline Setenta & \\
Ochenta & 7,2 \\
Noventa & 8,1 \\
Dosmil & 7,7 \\
Dos mil tres & 6,5 \\
\hline
\end{tabular}

Fuente: Cámara de Comercio de Neiva 2004.

exterior cuanto de un ingreso desbordado de divisas que revaluó el peso colombiano y abarató las importaciones.

Es pertinente recordar que las empresas que entraron en quiebra y en procesos concordatarios fue tan elevado que el Gobierno tuvo que proponer la ley 550 de 1999 de reactivación empresarial, permitiendo el debate sobre la formulación de nuevas estrategias e iniciativas que respondieran y ayudaran a sobrellevar los numerosos inconvenientes afrontados por la sociedad colombiana, la huilense y por supuesto la neivana. Precisamente frente a lo anterior, North (1995) plantea que

\section{Coeficiente de mortalidad/Natalidad en empresas de Neiva}

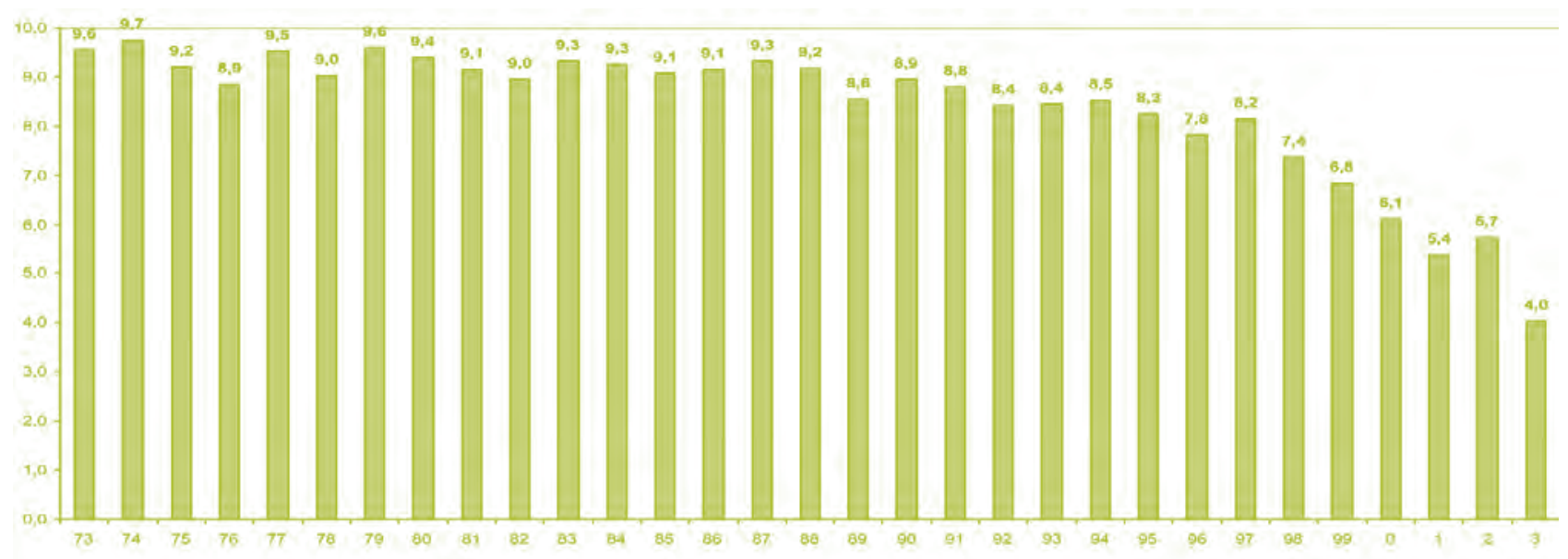

El coeficiente refleja cuantas empresas SALEN del mercado por cada 10 que ENTRAN en el mismo año.

Gráfica 2. Coeficiente de Mortalidad en empresas de Neiva $(H)$.

4. El desempeño del PIB de Colombia: 5,2\% en 1995, 2,1\% en 1996, 3,4\% en 1997, 0,6\% en 1998 y -4,2\% en el año 1999; 2,9\% en el 2000; 1,4\% en el 2001; 1,5\% en 2002: 3,7\% en 2003; 3,8\% en 2004 y 4\% en 2005. (DANE-Departamento nacional de Planeación 1995-2002).

5. El desempleo que en 1990 era de $10 \%$ llegó a niveles del $20 \%$ en el 2000 y aunque ha venido disminuyendo la tasa sigue siendo alta $13 \%$. Elaboración propia de los investigadores iniciales. 2007. 
la combinación de la teoría humana con las teoría de los costos de negociación fundamenta su teoría de las instituciones la cual permiten entender por qué existen las instituciones y su papel en la sociedad; basados en esta teoría Días Hernández \& Urbano (2005) enfatizan en la necesidad de las instituciones a proyectar reglas de juego claras y permanentes en el tiempo para que los empresarios denominados jugadores sean influenciados de manera positiva en la evolución económica de un país o región. En cambio, Julien (2008) propuso la idea del papel complementario del estado en facilitar el aumento de las capacidades de aprendizaje de los distintos actores que conforman el colectivo como son los dirigentes, empleados y empresarios, dinamizando la creación de redes de conocimiento procurando el fortalecimiento del desarrollo empresarial.

Por otra parte, Formichella (2004) afirma que a mayor educación en emprendimiento se aumenta la dotación social de cualidades emprendedoras logrando un mayor desarrollo y mejores niveles de educación local en la comunidad. A pesar de que la temática de emprendimiento se encuentra en las cátedras de los programas de pregrado, no se percibe que en ellos se aborden los factores que motivaron a los emprendedores del Huila a crear empresas. No se tiene una evidencia empírica acerca de las razones de esta conducta de los empresarios de la ciudad. Solo cuando se hace referencia al tema se mencionan ejemplos de otras latitudes y de empresas que no corresponden 0 identifican nuestra cultura e idiosincrasia. Es por ello, que se quiso determinar cuáles han sido los factores que han estimulado a los empresarios huilenses para crear empresa en el periodo 1973-2003.

En primer lugar, la necesidad de lograr unas mayores tasas de creación de empresas para compensar los efectos de la mortalidad y en segundo lugar mejorar el nivel de empleo y por ende, disminuir la pobreza en la ciudad y desacelerar tasa de mortalidad, debe ser un imperativo en nuestra sociedad. Para lograrlo es ineludible conocer bien los fenómenos tanto de creación de empresas como de mortalidad empresarial; de allí la urgencia de contribuir a proporcionar fundamentos para profundizar en el conocimiento de esta temática.

Se considera de alta conveniencia el desarrollo de esta investigación para la sociedad colombiana en general y huilense en particular, ya que el avance en la comprensión de los elementos que inciden en el proceso de creación de una nueva empresa permite disminuir el número de fracasos, haciendo más eficientes los esfuerzos emprendedores; además presenta un base que permite diseñar acciones, estrategias y políticas tanto del sector privado como público para el fortalecimiento del proceso de creación de organizaciones.

\section{Marco referencial y estado del arte}

Un documento a resaltar es el efectuado por Crissien (2008) donde presenta los empresarios de base universitaria EBU como factor importante en la creación de empresas; además expone propuesta de modelo de desarrollo económico basado en la educación empresarial como unidad generadora de tejido empresarial de calidad y de desarrollo económico, tomando como marco referencial la teoría institucional de North. Halló que en la educación empresarial en Colombia en la práctica no se adelanta ningún tipo de esfuerzos para educar o formar EBU. Finalizan proponiendo una clasificación de universidades con formación empresarial en niveles.

Otra experiencia importante de registrar es la elaborada por Lozano (2010) en su obra «La relación intrafamiliar en entornos empresariales. Incursión aun modelo de diagnóstico»; toma como base la información de 17 empresas familiares, para plantear un modelo que valore la relación intrafamiliar en familias propietarias de empresas, cuyo objetivo es precisar las variables de mayor impacto en las relaciones entre los miembros que componen el grupo familiar en entornos empresariales y de acuerdo con ello, intervenir sobre esas variables para beneficiar la armonía familiar, continuando con su empresa familiar.

En el texto titulado «El emprendedor y la empresa: una revisión teórica de los determinantes a su constitución», (Alonso Nuez \& Galve, 2008) se recapitula las principales teorías sobre creación de empresas existentes en la literatura y que más difusión han tenido, atendiendo a factores económicos, psicológicos, socioculturales e internos a la organización o empresa, Concluyen que la creación de una nueva empresa implica la intervención de diferentes actores, ya sean de carácter individual o corporativo, igualmente desde una perspectiva psicológica y social se ha asociado la conducta emprendedora con el predominio de valores individuales y también con la presencia de determinadas variables sociales dentro de los espacios familiar, laboral y personal. 
Se resalta los tipos de influencias sociales sobre el empresario potencial o efectivo de (Julien, 2008) las cuales son las afectivas (familia, amigos), simbólicas (educación, trabajo) y sociológicas (trabajo, experiencia y redes). Además, sobre los beneficios de las empresas familiares (Longenecker, Moore, \& Palich, 2008) están el fortalecimiento de los lazos familiares, se da mayor motivación interna y menos necesidad de recibir incentivos adicionales por parte de sus directores; además los recursos obtenidos suplen las necesidades familiares.

En documento de (Ebrashi, R.E, 2013) presenta una teoría para el emprendimiento social basado en la integración de la literatura empresarial con una investigación empírica mundial llevado a cabo en los empresarios sociales utilizando la teoría fundamentada. La teoría del comportamiento del emprendimiento social estudia los factores contextuales que llevan a la creación social de riesgo, la dinámica de organización y estructuras subyacentes, y cómo estas tipologías de medir el impacto social, la movilización de recursos, y lograr un cambio social sostenible.

Con respecto a los emprendimientos familiares Littunen, H. 2003 pretende aclarar cuáles son los factores asociados a la fase operativa inicial y fundamental de las empresas familiares y no familiares finlandesas influyen en la capacidad de las empresas para sobrevivir durante los críticos primeros tres años de su existencia. Los resultados revelaron diferencias marcadas en los motivos individuales para la fundación de una empresa: en los propietarios de empresas familiares primó la presencia de factores situacionales negativos como factor más importante de motivación en la creación de un nuevo negocio.

De los anteriores consultados se colige una gran variedad de factores que hace necesario tener un fundamento teórico que fundamente y guíe esta investigación

\section{Metodología de investigación}

Los factores analizados se hicieron con base en la orientación del modelo de Gibb, (1994) en los compo- nentes del modelo de carrera empresarial, donde se analizó cuáles son los factores individuales, familiares, sociales y económicos. Se redactaron preguntas conducentes a indagar descriptivamente dichos factores. Dentro de esta carrera empresarial Gibb Dayer Jr. ${ }^{6}$ expresa que debe estar compuesta por cuatro elementos o sub teorías, como son la teoría de la selección de carrera, la teoría de la socialización de la carrera, la teoría de orientación de carrera y la teoría de avance de carrera.

Esta investigación se enfoca en la teoría de selección de carrera que hace énfasis en los antecedentes que influyeron en la decisión de las personas a ser empresarios, como son los factores individuales, sociales y económi$\cos$, que orientan a buscar independencia y que es realmente el fundamento de esta investigación. Este modelo cuenta con tres grupos de factores que parecen incidir en la decisión de llegar a ser empresario.

El primer grupo está determinado por los factores individuales investigaciones recientes han notado que los factores individuales por los cuales una persona llega a ser empresario no están solo relacionados con rasgos de la personalidad, sino también con otros factores como el «ancla de carrera» estrategia diseñada (Schein, 1996) ${ }^{7}$, la cual se define como el sueño o la visión que tiene cada individuo sobre su futuro no solo profesional, sino también personal en lo concerniente a motivaciones y necesidades, talentos y habilidades, valores personales que no se sacrificarían si nos viéramos forzados a hacer una elección.

El segundo grupo está establecido por los factores sociales que están directamente relacionados por fuerzas implantadas en la sociedad, tales como: ambientes deprimidos (Pobreza en la niñez y padres negligentes), observando que los empresarios crecieron en ambientes donde temas dominantes eran la deserción, la muerte y la negligencia. Ambientes animados: (padres empresarios), los hijos de estos padres empresarios parecen seguir con esta misma vocación. Ambientes familiares: (soporte familiar), parece que el apoyo familiar tanto financiero como psicológico es otro factor bien importante en la selección de carrera. Ambientes culturales: (modos de vida y costumbres). Se considera que la raza y la cultura pueden tener un

6. Profesor de dirección, Brigham Young University.

7. Profesor de la MIT Sloan School of Management, investigador en las áreas de cultura organizacional, desarrollo de la organización, procesos de consulta y dinámicas de la carrera, creó un sistema de preguntas que permite conocer cuál es su ancla de carrera, estimulando la reflexión dirigida a fortalecer actitudes y aptitudes. 
impacto significativo al adoptar el rol de empresario, de igual modo, el apoyo de la comunidad también es muy importante pues será esta la encargada de reconocer 0 generar el prestigio a quien es propietario de un negocio.

Discurriendo en los factores familiares es necesario establecer el factor familiar que más haya motivado al individuo a crear empresas. Los referentes complementarios para este factor son: Silva (2008) quien afirma que el contexto familiar es el principal elemento facilitador 0 inhibidor del futuro emprendedor, esto se complementa con lo afirmado por Cuervo, Ribeiro, Roig (2007), sobre la aversión al riesgo ya que este puede llegar a afectar las expectativas del futuro familiar, y lo afirmado Ogliastri (2011) sobre las empresas familiares están al servicio de la familia versus el salvaguardar a la empresa de algunos familiares. Las empresas de origen familiar son importantes debido al gran número de empleos que generan, evidenciando la relevancia económica y social de la empresa familiar como lo aseveran por Zubizarreta, J. y Sanz, S (2012). Además Ruiz, (2008) destaca el compromiso como fuerza inherente de las empresas familiares.

En cuanto al factor social es imperioso determinar los principales factores sociales, al momento de crear empresas. Alonso y Gálvez, (2008) registran la importancia del entorno que rodea a la nueva empresa. Silva (2008) afirma que las fuerzas externas que inciden en las actitudes emprendedoras, alguna de ellas son el contexto sociocultural, la cultura laboral y el contexto organizacional y de cómo la armonía del emprendedor con ellas garantiza la supervivencia del proyecto emprendido. Cuervo et al. (2008) afirman que los contextos sociales presentan muchas restricciones para los emprendedores, aunque también fijan las condiciones que crean ventanas de oportunidad. Por otra parte, Polo, (2009) habla de la necesidad de las empresas de adquirir un compromiso social además de prestar un buen servicio.

También se encuentran los factores económicos que poseen influencia en la decisión de crear empresas. Montoya, (2009) enfatiza la relación sistemática entre el nivel de desarrollo económico de un país y el nivel y el tipo de actividad emprendedora; es importante señalar los más motivadores en el momento de crear empresa y la expectativa económica que se espera satisfacer en el momento de su creación. Algunos de estos factores son las condiciones económicas por las que viene atravesando el país. Aquí se destacan variables como el auge económico y situación general de empleo concebido como el enfren- tamiento de problemas de desempleo y subempleo que se vivían al momento de tomar su decisión de crear empresa (ante una aceleración económica la persona pudo haber decidido crear empresa porque veía ahí prosperidad y perspectivas). Disponibilidad de recursos financieros y no financieros (apoyo de instituciones privadas o públicas en asesoría y capacitación administrativa y técnica, y la situación particular de acceso al empleo concebida como la posibilidad individual de poder conseguir o no empleo. Por otra parte, Urbano, D., Díaz, J.C. y Mogollón H. (2007) enfatizan en la importancia de que las instituciones establezcan reglas claras, permanentes y así propiciar ambientes idóneos del desarrollo empresarial. Silva (2008) presenta lo económico de la mano con lo político, ya que las directrices del estado señalan el marco que inhibe o estimula la aparición de nuevas empresas.

Se siguieron los siguientes aspectos Metodológicos: se aplicó una encuesta incluyendo los factores anteriormente mencionados con preguntas de selección múltiple que permitió evaluar el pensamiento del empresario frente a los objetivos planteados; fue aplicada directamente a los gerentes y/o propietarios de las empresas activas según su tamaño: microempresas, empresas pequeñas y medianas empresas. Esta investigación es de tipo descriptivo, no experimental, porque busca sistematizar la información a partir del análisis estadístico, datos cuantitativos, utilizando las técnicas descriptivas, detallando una situación objeto de indagación. El universo está conformado por 24.201 empresas activas creadas durante periodo 1973 al 2003, según datos suministrados por la Cámara de Comercio de Neiva. Ver Gráfica 3.

Para el diseño de la muestra se utilizó un muestreo estratificado. Tomando como referencia a Montaña J. (2009), se estratificó por año de creación de la empresa (estrato I = periodo 1973 a 1987, estrato II = periodo 1988 a 1996 y estrato III = periodo 1997 a 2003) con afijación

Gráfica 3. Número de empresas de Neiva creadas en el periodo 1973-2003 según tamaño por activos

\begin{tabular}{|lcccc|}
\hline Grandes & Medianas & Pequeñas & Micros & Total \\
\hline 25 & 94 & 533 & 23.549 & 24.201 \\
$0,1 \%$ & $0,4 \%$ & $2,2 \%$ & $97,3 \%$ & $100 \%$ \\
& & & \\
Fuente: Alexander Pastrana: Alexandra Parra, Kelly González, Martha Polanía, Lady \\
Gómez, Melba Rocío Mora. Base salario mínimo de 2004, \$381.500.
\end{tabular}

Fuente: Elaboración propia de los investigadores iníciales. 2007 según datos suministrados por la Cámara de comercio de Neiva. 
proporcional, teniendo en cuenta una confiabilidad del 95\% para un error máximo admitido en las estimaciones del 4,7. Las empresas participantes en la muestra fueron seleccionadas aleatoriamente.

Para el procesamiento estadístico de las encuesta se empleó el Software estadístico Statistical package for the Social Sciencies - SPSS - versión 16.0, la hoja electrónica Excel y el procesador de palabras Word para elaborar informe final del documento.

De acuerdo a las características de las variables se emplearon las siguientes técnicas de estadística descriptiva, para resumir y analizar información: distribución de frecuencias, Cuadros de doble entrada, gráficos de sectores y barras utilizadas para graficar la información generada y visualizar de forma rápida un mayor entendimiento de la información ya agrupada. Se analizaron directamente variables cruzadas para analizar posibles relaciones 0 comunalidades. Dentro del presente estudio, la población escogida fue empresas creadas en Neiva en los periodos de tiempo ya mencionados en la explicación del Universo.

Esta estrategia de subdivisión, se hizo con el fin de manejar la información de acuerdo a las características socioeconómicas de la época y mirar de alguna forma si existía alguna correlación al final del presente estudio pues, abisma en la lectura de los informativos de dichos años, una luz que permite inferir la existencia de alguna correlación.

Sin embargo se encuentra que no hubo ninguna relación común entre factores de creación, descartando la idea inicial. Ver Cuadro 2.

\section{Resultados}

Dentro de los agentes estudiados se encuentran de manera general que las categorías más sobresaliente en orden de importancia en los factores individuales fueron: La necesidad de Ofrecer un mejor futuro a su descendencia, como preocupación prioritaria dentro de la mente del empresario huilense, la cual se encuentra en la categoría otros factores de crear empresas. Seguidamente en la categoría Factores valioso» para crear empresas se observa como aspecto sobresaliente las Habilidades para organizar la Experiencia adquirida previamente, lo anterior hace inferir que este factor individual que hace alusión conocimiento de origen empírico brindó al empresario seguridad en el desarrollo de actividad económica.

En la clase Expectativas en el momento de crear empresas sobresale, Liderazgo, Crecimiento profesional y Aprendizaje de nuevas habilidades; en la categoría Motivación a crear empresas para mejorar sus aspectos económicos se resaltan muy a la parla motivación de Necesidad de hacer cambios en su vida y Mejorar sus ingresos económicos. Ver Cuadro 3.

Dentro de los factores familiares valiosos para crear empresas, como aspecto a resaltar Deseos de trabajar con miembros de su familia y Presión familiar por producir recursos, en ese orden de importancia. Ver Cuadro 4.

En cuanto a los factores sociales encontramos que la mayor preocupación o necesidad socialmente vista por el empresario, en el momento de crear empresa, se encuentra ubicada en la categoría «Motivación para crear

Cuadro 2. Total muestra de empresas por periodo de estudio

\section{Total de empresas por periodo}

\begin{tabular}{lccc|}
\hline Tamaño & $1973-1987$ & $1988-1996$ & $1997-2003$ \\
\hline Microempresas & 18 & 31 & 31 \\
Pequeñas & 57 & 99 & 69 \\
Medianas & 37 & 194 & 0 \\
Subtotal & 112 & & 100 \\
Total muestra & & & 406 \\
\hline
\end{tabular}

Fuente: Elaboración propia de los investigadores VISIONARIOS, según datos suministrados por la Cámara de Comercio de Neiva. 
Cuadro 3. Factores individuales que influyeron en la creación de empresas en Neiva en el periodo comprendido entre 1973 a 2003

Selección de carrera Factores globales (Categorías)

\section{Micro Pequeña Mediana Promedio}

\section{Factores individuales Motivación a crear empresas}

Deseo a enfrentarse a retos

Necesidad de logros

Necesidad de hacer cambios en su vida (5)

Mejorar sus ingresos económicos (5)

Deseo de auto-gestionar el tiempo y destino personal

Totales

$\begin{array}{cccc}19 & 13 & 20 & 16 \\ 21 & 21 & 1 & 16 \\ 19 & 21 & 39 & 25 \\ 26 & 29 & 15 & 25 \\ 14 & 16 & 25 & 18 \\ 100 & 100 & 100 & 100\end{array}$

Factores valiosos para crear empresas

Habilidades para organizar (2)

Experiencia adquirida previamente (3)

Relaciones interpersonales

Placer por sr responsable en tareas o actividades

Otro-cuál

Totales

$\begin{array}{cccc}10 & 34 & 74 & 39 \\ 45 & 29 & 26 & 31 \\ 10 & 27 & 0 & 17 \\ 24 & 10 & 0 & 10 \\ 11 & 0 & 0 & 2 \\ 100 & 100 & 100 & 100\end{array}$

\section{Expectactivas en el momento de crear empresas}

Crecimiento profesional

Liderazgo (4)

Aprendizaje de nuevas habilidades

Posibilidad de controlar su tiempo

Desarrollo de autoestima

Totales

$\begin{array}{cccc}25 & 20 & 23 & 22 \\ 22 & 29 & 29 & 28 \\ 19 & 20 & 25 & 21 \\ 18 & 21 & 13 & 18 \\ 4 & 11 & 9 & 9 \\ 100 & 100 & 100 & 100\end{array}$

Otros factores individuales que expresan el deseo en el momento de crear empresas

Lograr ascender a un nivel social superior

$24 \quad 28 \quad 0$

Ofrecer un mejor futuro a su descendencia (1)

Ser conocido en la comunidad a la que pertenece

Otro-cuál

Ninguno

$\begin{array}{llll}61 & 56 & 100 & 68\end{array}$

Totales

$3 \quad 8$

$0 \quad 3 \quad 0$

$2 \quad 5 \quad 0$

$100 \quad 100 \quad 100 \quad 100$

Cuadro 4. Factores Familiares que influyeron en la creación de empresas en Neiva en el periodo comprendido entre 1973 a 2003

Selección de carrera Factores globales (Categorías)

Micro Pequeña Mediana Promedio

\section{Factores familiares Motivación a crear empresas}

Deseo de trabajar con miembros de su familia (1) $\quad \begin{array}{llll}53 & 32 & 23 & 34\end{array}$

Tradición familiar de creación de empresas

$\begin{array}{cccc}53 & 32 & 23 & 34 \\ 21 & 9 & 10 & 12 \\ 13 & 30 & 40 & 29 \\ 13 & 29 & 27 & 25 \\ 0 & 29 & 0 & 0 \\ 100 & 100 & 100 & 100\end{array}$

Presión familiar por producir recursos (2)

Rompimiento de la dependencia de la familia

Ninguno 
empresas» fue el «Ofrecer un mejor futuro a su descendencia»; seguidamente observamos dentro de la categoría Factores valiosos para crear empresas sobresale en orden de importancia «Voluntad de prestar un servicio a su comunidad» como dato considerado moda dentro del estudio. Ver Cuadro 5.

Y por último dentro de los «Factores económicos» se observa que en la categoría «Con potencial y desarrollo en el tiempo»s se encuentra el factor global con el promedio general de mayor incidencia el cual fue «Preparación académica enfocada a la cultura emprendedora». Le siguen en la categoría «Motivación a crear empresas» el factor: «Identificación de una oportunidad en el mercado» y en la categoría «Expectativas económicas en el momento de crear empresas», el factor de «Asegurar su futuro» con igual promedio. Los factores en tercer y cuarto lugar de importancia son «Deseo de mayores ingresos» e «incentivos tributarios». Ver Cuadro 6.

En la categoría «Con potencial de desarrollo y permanencia en el tiempo, el factor «La Preparación académica enfocada a la cultura emprendedora» se destaca de los otros factores; esta semilla sembrada en la mente de los empresarios neivanos germinó y su fruto fue la creación de una empresa. «Los Incentivos Tributarios», fue un factor especialmente importante para la pequeña empresa; cabe hacer énfasis que la calificación más alta se observa durante el año 1995, (dentro del período de 1997 a 2003), basado muy seguramente en los incentivos otorgados a los inversionistas que se acogieron (Congreso de la República de Colombia, 1995) la ley 218 de 1995 (Ley Páez) y sus respectivos decretos reglamentarios. La «Facilidad de acceso a recursos financieros», fue factor predominante para la creación de las microempresas en el consolidado del estudio. Por otra parte los factores «La seguridad en el orden público», «Auxilios y asistencia gubernamental «no son determinantes en esta categoría.

\section{Conclusiones}

De lo anterior se infiere que los emprendedores de la ciudad de Neiva, en el período de tiempo estudiado, años 1973 a 2003; dentro los «factores individuales», o sea aquellos intrínsecos dentro del ser humano, en su condición individual, en orden de importancia fueron el Deseo de Ofrecer un mejor futuro a su descendencia, habilidades para organizar Experiencia adquirida previamente, esto dentro del desarrollo de su vida laboral y personal.

En los factores familiares la categoría con mayor promedio fue El deseo de trabajar con miembros de su familia, seguida de la Presión familiar por producir recursos, ambas

Cuadro 5. Factores sociales que influyeron en la creación de empresas en Neiva en el periodo comprendido entre 1973 a 2003

\section{Selección de carrera Factores globales Micro Pequeña Mediana Promedio}

\begin{tabular}{|c|c|c|c|c|c|}
\hline \multirow[t]{13}{*}{ Factores sociales } & \multicolumn{5}{|l|}{ Motivación a crear empresas } \\
\hline & Voluntad de prestar un servicio a la comunidad (2) & 56 & 70 & 74 & 68 \\
\hline & Convertirse en solución de empleo para un sector social & 26 & 30 & 0 & 22 \\
\hline & Contribuir a mejorar condiciones sociales de algún grupo & 15 & 0 & 0 & 3 \\
\hline & Contribuir responsablemente con el cuidado del medio ambiente & 3 & 0 & 0 & 1 \\
\hline & Otro - cuál & 0 & 0 & 26 & 6 \\
\hline & Totales & 100 & 100 & 100 & 100 \\
\hline & \multicolumn{5}{|l|}{ Factores valiosos para crear empresas } \\
\hline & Lograr ascender a u n nivel social superior & 21 & 27 & 0 & 19 \\
\hline & Ofrecer un mejor futuro a su descendencia (1) & 64 & 61 & 97 & 71 \\
\hline & Ser reconocido dentro de la comunidad a que pertenece & 15 & 12 & 0 & 10 \\
\hline & Otro-cual & 0 & 0 & 3 & 1 \\
\hline & Totales & 100 & 100 & 100 & 100 \\
\hline
\end{tabular}


Cuadro 6. Factores Económicos que influyeron en la creación de empresas en Neiva en el periodo comprendido entre 1973 a 2003

\begin{tabular}{|c|c|c|c|c|c|}
\hline Selección de carrera & Factores globales (Categorías) & Micro & Pequeña & Mediana & Promedio \\
\hline \multirow[t]{21}{*}{ Factores económicos } & \multicolumn{5}{|l|}{ Motivación a crear empresas } \\
\hline & Identificación de una oportunidad en el mercado (2) & 17 & 46 & 74 & 47 \\
\hline & Deseo de mayores ingresos ( 3 ) & 48 & 39 & 26 & 38 \\
\hline & Alternativa al desempleo & 35 & 15 & 0 & 15 \\
\hline & Otro-cuál & 0 & 0 & 0 & 0 \\
\hline & Totales & 100 & 100 & 100 & 100 \\
\hline & \multicolumn{5}{|l|}{ Expectativas económicas en el momento de crear empresas } \\
\hline & Independencia & 45 & 22 & 0 & 21 \\
\hline & Asegurar su futuro (1) & 37 & 27 & 100 & 47 \\
\hline & Conseguir los medios para realizar otros proyectos & 15 & 15 & 0 & 11 \\
\hline & Autonomía financiera & 3 & 36 & 0 & 21 \\
\hline & Otro-cuál & 0 & 0 & 0 & 0 \\
\hline & Totales & 100 & 100 & 100 & 100 \\
\hline & \multicolumn{5}{|l|}{ Con potencial de desarrollo y permanencia en el tiempo } \\
\hline & Incentivos tributarios & 14 & 50 & 26 & 37 \\
\hline & Facilidad de acceso a recursos financieros & 52 & 0 & 0 & 10 \\
\hline & Seguridad en el orden público & 2 & 0 & 0 & 0 \\
\hline & Auxilios o asistencia gubernamental & 5 & 0 & 0 & 1 \\
\hline & \multicolumn{5}{|l|}{ Preparación académica enfocada a la cultura } \\
\hline & emprendedora(1) & 28 & 0 & 0 & 52 \\
\hline & Totales & 100 & 100 & 100 & 100 \\
\hline
\end{tabular}

categorías reflejan la importancia de la familia para el emprendedor Huilense.

En cuanto a los factores sociales, se encontró que la mayor preocupación o necesidad vista por el empresario en el momento de crear empresa fue Ofrecer un mejor futuro a su descendencia seguido de la Voluntad de prestar un servicio a su comunidad observada esta última categoría como una moda dentro de la dispersión de punto, lo cual permite aseverar que fue de manera indiscutible como categoría única.

Desde el punto de vista de los Factores económicos se observó que la categoría reparación académica enfocada a una cultura emprendedora fue la que alcanzó el mayor promedio; esto pone de presente la importancia que tiene la academia frente a estos procesos de emprendimiento que se generan en la región huilense. Con igual promedio se encuentran Identificación de una oportunidad de negocio y Asegurar su futuro. Esto debería ser una prioridad en la redacción de los currículos académicos, en asignaturas y conferencias, pues la facilidad de encontrar esas oportunidades favorecerá la creación de empresas. El Deseo de mejorar ingresos en segundo lugar y en tercer lugar Incentivos tributarios, que tuvo en cuenta el empresario para poder desarrollar su idea de creación empresarial. Esta situación se presenta especialmente en la fecha en que existió la ley 218 de 1995 (Ley Páez) en Neiva donde la calificación se notó alta en el año 1995 , dentro del período de 1997 a 2003, basado muy seguramente en los incentivos otorgados a los inversionistas que se acogieron a la ley y sus respectivos decretos reglamentarios. Ya lo presentaba North (1995) retomado por Urbano, D., Díaz, J.C. y Mogollón H. (2007), en lo importante de que las instituciones gubernamentales establezcan «reglas de juego» claras y permanentes para que esto se vea reflejado en la actividades efectuadas por los empresarios impactando de manera positiva en el desarrollo de un país o región. 


\section{Referencias bibliográficas}

Alonso M, Galve C. El emprendedor y la empresa: una revisión teórica de los determinantes a su constitución. Acciones e investigaciones sociales. Zaragosa: Departamento de economía y dirección de empresas, 2008.

Colmenares J. Colombia: crisis del crecimiento económico: Expresión en el comportamiento de la oferta final 1990-2002. Rev Economía y desarrollo, 2(3)(2003).

Congreso de la República de Colombia. Ley 218 de 1995. Diario Oficial, No. 42.117, de 22 de noviembre de 1995 Por la cual se modifica el Decreto 1264 del 21 de junio de 1994 proferido en desarrollo de la emergencia declarada mediante Decreto 1178 del 9 de junio de 1994 y se dictan otras disposiciones. Recuperado el 23 de junio de 2012.

Congreso de la República de Colombia. «Ley 550 de 1999». Por la cual se establece un régimen que promueva y facilite la reactivación empresarial y la reestructuración de los entes, 1999.

Crissien, J. O. Círculo virtuoso de la educación empresarial: una propuesta de modelo para el desarrollo de un tejido empresarial de calidad. Revista Escuela de administración de Negocios, 63(2008):23-39.

Cuervo Á, Ribeiro D, \& Roig, S. Entrepreneurship: conceptos, teoría y perspectiva. Valencia: Editorial Fundación Bancaja, 2007.

Ebrashi, R.E, «Social entrepreneurship theory and sustainable social impact». Rev «http://siaa.ucc.edu. co:2057/source/sourcelnfo.url?sourceld=19900191756 \&origin=recordpage» 10 «Go to the information page for this source» Social Responsibility Journal, 9(Issue 2) (2013):188-209.

Formichella M. El concepto del emprendimiento y su relación con al educación, el empleo y el desarrollo local». Instituto Nacional de tecnología agropecuaria. Convenio INTA - Ministerio de Asuntos Agrarios y Producción - Pcia. de Buenos Aires. Chacra Experimental Integrada Barrow, 2004.

Gartner W. A conceptual framework for describing the phenomenon of new venture creation academy of management review. 1985. Academy of managment Riew, 10(4).
Gibb Dayer Jr., W. Toward a theory of entrepreneurial careers, entrepreneurship theory and practice. Entrepreneurship: Theory and Practice, 19(2)(1994).

Gobernación del Huila. «Anuario estadístico del Huila». Neiva, Departamento Administrativo de Planeación, 65, 89, 2003.

Julien, P. Emprendimiento regional y economía del conocimiento. Santiago de Cali: Pontificia Universidad Javeriana de Cali, p. 103, 317-308, 2008.

Leivobich, J. «Los desafíos institucionales para reducir la pobreza en Colombia, Seminario medición de la pobreza y estrategias para reducirla», 2005.

Llopis, F. Iniciativa empresarial de la pequeña empresa: un análisis de la situación actual. Alicante: Digital, 2008.

Longenecker J, Moore, C., \& Palich, L. Administración de pequeñas empresas. México: CENGAGE Learning, 2008.

Lozano M. La relación intrafamiliar en entornos empresariales. Incursión a un modelo de diagnóstico. Rev Estudios gerenciales, 22(100)(2006):125-151 . «http:// siaa.ucc.edu.co:2068/indexinglinkhandler/sng/au/ Littunen, + Hannu/\$N?accountid=44394» ।

Littunen, H. Management capabilities and environmental characteristics in the critical operational phase of entrepreneurship-a comparison of Finnish family and nonfamily firms. Rev «http://siaa.ucc.edu.co:2068/pubid linkhandler/sng/pubtitle/Family+Business+Review/\$N/ 36981/DocView/211109606/fulltextwithgraphics/14131 FA04876E12B157/1?accountid=44394»|Family Business Review HYPERLINK «http://siaa.ucc.edu.co: 2068/indexin gvolumeissuelinkhandler/36981/Family+Business+ Review/02003Y09Y01\$23Sep+2003 \$3b++Vol.+16+ $\$ 283 \$ 29 / 16 / 3$ ?accountid=44394» $\backslash 16.3$ p. 183-197.2003.

Montaño, J. «Estadística inferencial para el sector industrial». Neiva: Universidad Surcolombiana, 2009.

Montoya, L. La creación de spin-offs de base académica - investigativa por iniciativa de los estudiantes: el caso de la Universidad Nacional de Colombia sede Medellín. Tesis de Maestría. Medellín. Ingeniería Administrativa, Facultad de Minas, Universidad Nacional de Colombia. 
Morales S, Roig S. The New Venture Decision: An Analysis Based. Rev International Entrepreneurship and Management Journal 1, 479-499. Universitat de València, 2005.

North, D. Instituciones, cambio institucional y desempeño económico». Rev Fondo de cultura económica, 188, 1995.

Ogliastri, E. «Empresa familiar emprendedora». Rev INCAE Business Review. Volumen 2 / Número 2 / Mayo agosto, 3, 2011.

Pallares D, Romero D, y Herrera, M. «Hacer Empresa: Un reto». (Cuarta ed.). Bogotá: Fondo editorial nueva empresa, 2011.

Polo, J. La responsabilidad social corporativa de las grandes empresas españolas con presencia en América Latina: modelos de actuación. Madrid: Universidad Complutense de Madrid, 2011.

Ruiz, M. Gestión de la empresa familiar. España: McGraw-Hill, 2008.

Schein, E. Career anchors revised: Implications for career development in the 21st century. Recuperado el 10 de 10 de 2012, de The Academy of Management Executive, 1996.
Silva, J. «Emprendedor, crear su propia empresa». Colombia, Bogotá, Colombia: Alfaomega Colombiana, S.A., 2008.

Toledano N, Urbano D. Invitación al emprendimiento: una aproximación a la creación de empresas. España: Editorial UOC., 2008.

Urbano D, Díaz C, Mogollón H. «Evolución y principios de la teoría económica institucional. Una propuesta de aplicación para el análisis de los factores condicionantes de la creación de empresas». En Investigaciones Europeas de Dirección y Economía de la Empresa. 13(3)(2007): 183-198.

Varela R. Innovación empresarial, arte y ciencia en la creación de empresas. Bogotá: Pretince Hall, 2001.

Zubizarreta J, Sanz S. La empresa familiar y el emprendimiento». En: «http://siaa.ucc.edu.co:2068/pubidlink handler/sng/pubtitle/Bolet\$edn+de+Estudios+Econ \$f3micos/\$N/1536340/DocView/1318921886/fulltext/ 1412E4AF63B11C70121/2?accountid=44394» lo Boletín de Estudios Económicos «http://siaa.ucc.edu.co:2068/ indexingvolumeissuelinkhandler/1536340/Bolet\$edn+de+ Estudios+Econ\$3micos/02012Y04Y01\$23Apr+ 2012 $\$ 3 b++$ Vol. $+67+\$ 28205 \$ 29 / 67 / 205$ ? accountid=44394»lo «Hacer clic para buscar más entradas de este número» 67.205, (2012):145-160. 